\title{
Prevalence and Etiologic Agents of Dermatophytosis among Primary School Children in Harari Regional State, Ethiopia
}

\author{
Alem Alemayehu, ${ }^{1}$ Gebremedhin Minwuyelet, ${ }^{2}$ and Gizachew Andualem ${ }^{1}$ \\ ${ }^{1}$ Department of Medical Laboratory Science, College of Health and Medical Sciences, Haramaya University, \\ P.O. Box 235, Harar, Ethiopia \\ ${ }^{2}$ Department of Medicine, College of Health and Medical Sciences, Haramaya University, P.O. Box 235, Harar, Ethiopia
}

Correspondence should be addressed to Alem Alemayehu; gechayu2006@gmail.com

Received 16 May 2016; Accepted 18 July 2016

Academic Editor: Simona Nardoni

\begin{abstract}
Copyright (C) 2016 Alem Alemayehu et al. This is an open access article distributed under the Creative Commons Attribution License, which permits unrestricted use, distribution, and reproduction in any medium, provided the original work is properly cited.

Dermatophytes are worldwide in distribution and dermatophytosis is a common problem in developing countries. It can occur in both sexes and all ages but the diseases are more common in school children. This study attempted to determine the prevalence and etiological agents of dermatophyte infections of hair, skin, and nail among primary school children in Harari Regional State from April to June 2015. A cross-sectional study was conducted on 428 primary school children. Skin scrapings, hair samples, and nail clippings were collected from children who showed dermatophytosis. All specimens were subjected to microscopic examination and culture. Following a meticulous collection, data was analysed using SPSS version 21 . Of the 428 school children, 211 (49\%) male and 217 (51\%) female, 100 (23.4\%) had culture confirmed dermatophytosis and tinea capitis took the overall prevalence of $18 \%$ (77/428). Trichophyton violaceum was isolated from 43 samples, followed by Trichophyton rubrum in 24 . The highest prevalence of dermatophytosis was seen in the age group 5-9 years and grade levels of 1-2 $(p<0.05)$. As a result, this study found a high prevalence of dermatophytosis in the Harari's Regional State school children and tinea capitis was the predominant clinical finding which needs an intervention.
\end{abstract}

\section{Introduction}

Fungal infections of the skin, hair, and nails due to dermatophytes are a common problem across the globe. In 2005, the overall incidence of tinea in sub-Saharan Africa was estimated to be 78 million $[1,2]$. Depending on the climate and culture, the clinical picture can differ. Tinea pedis and onychomycosis are the most prevalent clinical forms in western countries, while tinea capitis and tinea corporis are the most frequent forms in tropical areas $[1,3]$. Individuals of both sexes and all ages are susceptible to dermatophytoses; however, tinea capitis is more common in prepubescent children, and tinea cruris and tinea pedis are primarily diseases of adult males [2]. Tinea capitis is also the most prevalent clinical form in African younger age population $[3,4]$.

There are approximately 40 different species of dermatophytes, of which the most common species that cause disease in human are Trichophyton rubrum, Trichophyton tonsurans, Trichophyton interdigitale, Trichophyton mentagrophytes, Microsporum canis, and Epidermophyton floccosum. On a worldwide scale, T. rubrum and T. mentagrophytes account together for $80 \%$ to $90 \%$ of all dermatophytosis $[1,5,6]$.

Although dermatophytosis occurs worldwide, individual dermatophyte species may vary in their geographic distribution and self-virulence. These disorders cannot be differentiated by ethnicity or socioeconomic status, but poverty and overcrowded living conditions are important underlying social determinants [7]. Factors contributing to the high frequency and chronic occurrences of dermatophytosis in developing countries may also include poor living conditions, children interaction patterns, and poor health seeking behaviour [8].

The infection is very common among children and people who have pets, have wet skin condition, have skin injuries 
or abrasions, use public showers, are barefoot, and share hairbrushes or unwashed clothing with other people. Those have an increased risk of developing the infection $[1,5,9]$.

It is difficult to ascertain reliably the overall incidence and prevalence of the various cutaneous infections in different parts of the world because studies of one region of the country may not be a true representation of the overall disease pattern of that country; furthermore, incidence and prevalence figures may only be representative of the population sampled, which may have associated risk factors for infection.

Few studies have investigated the etiology of cutaneous fungal infections in the developing world, and, consequently, there is less knowledge of any changes to their epidemiology. Knowledge of the predominant causative species provides a clearer understanding of risk factors for superficial fungal infections and future epidemiologic trends [5]. Like many other areas of the country, there is no research done on dermatophyte infections in Harari Regional State, Ethiopia. So this research was conducted to determine the etiological agents, clinical forms, and prevalence of dermatophyte infection in the region.

\section{Materials and Methods}

A cross-sectional study was conducted from April to June 2015. A multistage random sampling technique was used to select 428 students from nine primary schools. Clinical and laboratory data collection format which includes the children's age, sex, grade level, and residence was used to collect the required information.

2.1. Ethical Clearance. The study was approved by the Ethical Committee of the College of Health and Medical Science, Haramaya University. A letter was written for all concerned officials; permission was obtained from Harari region education bureau, Woreda education office, and school administrators. Informed written consent was obtained from school principals. Then, each study participant was informed about the objectives of the study, and verbal assent was obtained from the children.

2.2. Clinical Examination. Children were examined in natural day light by a dermatologist for visible clinical signs (erythema, alopecia, scaling, crusting, circinate lesions or follicular inflammation, pruritus, etc.) of dermatophyte infection.

2.3. Microbiological Analysis. Scrapings from the skin consisting of epidermal scales and from the infected hairs were taken using a sterile scalpel blade on a piece of black paper and tooth brush, respectively, after cleaning the affected sites with $70 \mathrm{v} / \mathrm{v}$ alcohol. Cotton swabs were used to collect pus from inflammatory lesions.

Each specimen was appropriately labelled and subsequently transported within 24 hours for microscopic examination and culture. A portion of each sample was examined microscopically by $\mathrm{KOH} 10-20 \%$ and lactophenol cotton blue solutions. The other portion was cultivated on Sabouraud's dextrose agar. The cultures were incubated at $25^{\circ} \mathrm{C}-30^{\circ} \mathrm{C}$ for up to four weeks. Identification of the etiological agents was
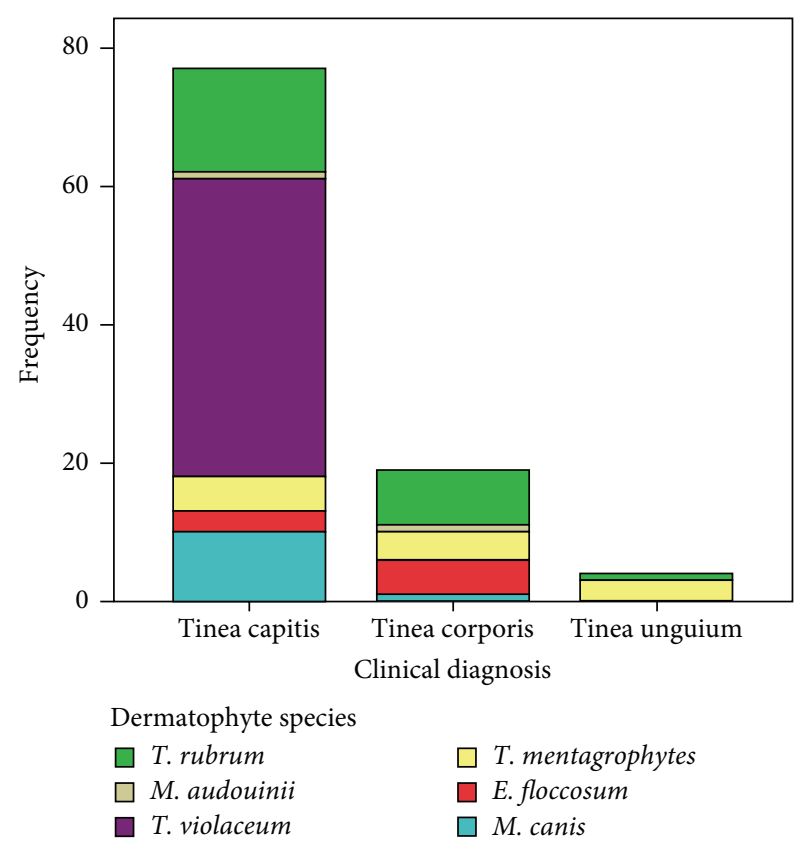

FIgure 1: Clinical presentation of dermatophytosis and their etiologic agents among primary school children in Harari Regional State, Ethiopia, from April to June 2015.

performed based on the gross morphology of the fungal colony, rate of colony growth, and microscopic characterization of their conidia and hyphae [10].

2.4. Statistical Analysis. Data was entered, cleaned, and analysed by using SPSS version 21 statistical package. Descriptive statistical tests are computed for categorical and continuous variables. Chi-square was calculated and $p$ value $<0.05$ was considered statistically significant.

\section{Results}

Four hundred twenty-eight children participated from nine different primary schools which are found in Harari Regional State including the city and its suburb. From these study subjects 211 (49\%) were male and 217 (51\%) were female with mean ( \pm sd) age $11 \pm 2$. Most of the school children were (67\%) in the age ranges of 10-14 years; significant portion of the study subjects were from the urban settlement (97\%) within the public school (87\%).

Out of the 428 school children, 115 (26.8\%) were clinically suspected to have dermatophyte infection. Among those clinically diagnosed patients, the laboratory culture confirmed cases were 100 (23\%). More than one type of clinical presentation was seen in over $3 \%$ of the children.

From the 131 clinical samples that were collected from 115 school children, 123 (93.8\%) had fungal elements under microscope examination (KOH and lactophenol cotton blue) and 100 of them (76\%) had growth on culture media. As shown in Figure 1, tinea capitis took the overall prevalence of 77 culture confirmed cases. T. violaceum were recovered from 43 samples, followed by T. rubrum in 24 . 
TABLE 1: Frequency distribution of dermatophytosis with respect to students' sex, age, and residence in Harari Regional State, Ethiopia, from April to June 2015.

\begin{tabular}{|c|c|c|c|c|c|c|c|c|c|c|c|c|c|c|c|}
\hline \multirow{2}{*}{\multicolumn{2}{|c|}{ Clinical diagnosis }} & \multicolumn{2}{|c|}{ Sex } & \multirow{2}{*}{$X^{2}$} & \multicolumn{2}{|c|}{ Residence } & \multirow{2}{*}{$X^{2}$} & \multicolumn{3}{|c|}{ Age group } & \multirow{2}{*}{$X^{2}$} & \multicolumn{3}{|c|}{ Grade level } & \multirow{2}{*}{$X^{2}$} \\
\hline & & Male & Female & & Urban & Rural & & $5-9$ & $10-14$ & $\geq 15$ & & $1-2$ & $3-4$ & $5-6$ & \\
\hline \multirow{5}{*}{ Positive } & Tinea capitis & 39 & 38 & \multirow{5}{*}{0.000} & 70 & 7 & \multirow{5}{*}{0.086} & 32 & 45 & 0 & \multirow{5}{*}{0.012} & 34 & 24 & 19 & \multirow{5}{*}{0.004} \\
\hline & Tinea corporis & 9 & 9 & & 17 & 1 & & 4 & 14 & 0 & & 6 & 2 & 10 & \\
\hline & Tinea unguium & 2 & 2 & & 4 & 0 & & 0 & 4 & 0 & & 0 & 0 & 4 & \\
\hline & Tinea capitis + tinea corporis & 9 & 6 & & 15 & 0 & & 7 & 8 & 0 & & 7 & 3 & 5 & \\
\hline & Tinea capitis + tinea unguium & 1 & 0 & & 1 & 0 & & 1 & 0 & 0 & & 1 & 0 & 0 & \\
\hline Negative & & 151 & 162 & 0.000 & 303 & 10 & 0.086 & 85 & 215 & 13 & 0.012 & 76 & 103 & 134 & 0.004 \\
\hline Total & & 211 & 217 & & 410 & 18 & & 129 & 286 & 13 & & 124 & 127 & 177 & \\
\hline
\end{tabular}

TABLE 2: Frequency distribution of dermatophytes with respect to sex, age, grade level, and residence in Harari Regional State, Ethiopia, from April to June 2015.

\begin{tabular}{|c|c|c|c|c|c|c|c|c|c|c|c|c|c|c|}
\hline \multirow{2}{*}{ Culture result } & \multirow{2}{*}{ Dermatophyte species } & \multicolumn{2}{|c|}{ Sex } & \multirow{2}{*}{$X^{2}$} & \multicolumn{2}{|c|}{ Residence } & \multirow{2}{*}{$X^{2}$} & \multicolumn{2}{|c|}{ Age group } & \multirow{2}{*}{$X^{2}$} & \multicolumn{3}{|c|}{ Grade levels } & \multirow{2}{*}{$X^{2}$} \\
\hline & & Male & Female & & Urban & Rural & & $5-9$ & $10-14$ & & $1-2$ & $3-4$ & $5-6$ & \\
\hline \multirow{6}{*}{ Positive 100} & T. rubrum & 14 & 10 & \multirow{6}{*}{0.034} & 24 & 0 & \multirow{6}{*}{0.047} & 11 & 13 & \multirow{6}{*}{0.843} & 12 & 4 & 8 & \multirow{6}{*}{0.306} \\
\hline & M. audouinii & 0 & 2 & & 2 & 0 & & 1 & 1 & & 1 & 1 & 0 & \\
\hline & T. violaceum & 25 & 18 & & 41 & 2 & & 15 & 28 & & 16 & 11 & 16 & \\
\hline & T. mentagrophytes & 7 & 5 & & 11 & 1 & & 3 & 9 & & 6 & 0 & 6 & \\
\hline & E. floccosum & 0 & 8 & & 8 & 0 & & 3 & 5 & & 3 & 4 & 1 & \\
\hline & M. canis & 6 & 5 & & 8 & 33 & & 5 & 6 & & 5 & 1 & 5 & \\
\hline Negative & 15 & 8 & 7 & & 13 & 2 & & 6 & 9 & & 5 & 3 & 7 & \\
\hline Total & 115 & 60 & 55 & & 107 & 8 & & 44 & 71 & & 48 & 24 & 43 & \\
\hline
\end{tabular}

The prevalence of dermatophyte infection has variable frequencies with respect to the residence and gender grouping of participants, but there was not a statistically significant association ( $p>0.05)$. However, there was a statistically significant association between dermatophytosis prevalence and the students' sex and age group as well as between grade levels $(p>0.05)$ as shown in Table 1 . The highest prevalence was seen in age group 11-14 and grade levels 1-2 and the least was in children age $\geq 15$ and grades 5-6, respectively.

Regarding etiologic agents, 100 dermatophytes were isolated from 115 clinically suspected school children. There were frequency variations with respect to age, sex, grade level, and residence as summarised in Table 2. T. violaceum (38.3\%) and T. rubrum (22.4\%) have had a higher frequency in urban residents whereas $M$. canis was the most common dermatophyte species in the rural school children $(37.5 \%)$. Concerning grade level, T. rubrum (25\%) and T. mentagrophytes (12\%) were more prevalent in grade levels 1-2.

\section{Discussion}

As confirmed by different studies, the prevalence and etiologic agents of dermatophytic infection vary depending on geographical location, socioeconomic status, time, and life style of the studied population. School children, the study subjects, are particularly potential victims of dermatophytic fungal infection either due to age related physiological conditions or due to their level of hygiene practice. Further, they are prone to acquire the infection due to their school setup and hence the conducive situation of passing the disease on to another susceptible hosts. The objective of this study was to determine the prevalence and etiological agents of dermatophytosis in the region.

The prevalence of dermatophytosis was $23.4 \%$ in the current study whereas in other similar studies which were done in Kenya, Nigeria, Tanzania, and Spain on primary school children it was $5.0 \%, 31.6 \%, 11.4 \%$, and $2.9 \%$, respectively [1114]. These variations between countries show that the prevalence of dermatophytic fungal infection varies depending on climate condition, socioeconomic status, and geographical location of the studied population.

This study also found that tinea capitis was the predominant clinical presentation $77 / 115$ (66.6\%) which is similar to other studies done in African school children with $>50 \%$ prevalence $[12,15]$, but in Barcelona it accounts only for $8.8 \%$ (3/34); instead, tinea pedis was leading [14]. These indicate that life style, culture, economic status, and so forth may contribute to specific clinical presentation of dermatophyte infection in a given population.

Regarding the causative agents, $T$. violaceum was the predominant species that was isolated from 43/77 scalp samples, followed by T. rubrum 15/77 which was the frequent dermatophyte from skin scraps $8 / 31$ whereas in nail infection T. mentagrophytes was leading 3/4. In Nigeria, $M$. audouinii was the most common from scalp sample [15]; in Tanzania, M. canis was isolated from 14 samples followed by T. violaceum 6/30 [12]; in Barcelona of Spain, T. mentagrophytes was the most common species, being isolated 
in two cases of tinea capitis and in 16 feet samples out of 35 and T. violaceum in one case of tinea capitis in a child from Ethiopia [13]. These etiologic agent variations are due to the type of dermatophyte found in a particular area; life styles of the studied population like farming, having domestic animals, crowding, and migrating and study period could be responsible reasons.

In relation to that, the particular case of interest is the finding of this study with regard to the finding on an Ethiopian immigrant studied in Barcelona [13]; coincidentally, the etiologic agent was similar, that is, T. violaceum, which could be due to the fact that the child transported this species from Ethiopia to Barcelona and that asserts the logic which was deducted earlier with regard to the occurrence of a particular dermatophyte species.

This study also assessed the association of sex, age, residence, and grade level with dermatophytosis prevalence. Accordingly, increased prevalence of dermatophytosis was seen in male children at $28 \%$, age group $5-9$ years at $34.1 \%$, grade levels $1-2$ at $38.7 \%$, and rural residence at $44.4 \%$. There was a statistically significant association between sex, age group, and grade level $(p<0.05)$, which is similar to other studies done in Africa; in Tanzania, among pupils aged $6-10$ years, it was $21.8 \%(p=0.045)$ and without sex difference, and the prevalence in males and females was $12.6 \%$ and $10.1 \%$, respectively. In Nigeria, also dermatophytosis was significantly higher $(p<0.05)$ in children aged $7-11$ years [14]. The discordant result with other studies regarding sex is maybe due to the higher number of male students in the age group 5-9 years and 10-14 which had higher positivity rate. We could not find a study that assessed student's grade level but this study found an increased prevalence in grade levels $1-2$ with statistically significant association $(p=0.001)$. The reason was that student's grade level is related with age group and $71 \%$ of grade $1-2$ students found in 5-9 years age in this study.

An increased frequency of tinea capitis was seen in age group 5-9 years old $(30.2 \%)$ among rural residents $38.9 \%$ and grade levels 1-2 (32.3\%) without sex difference in the present study which is similar to another African study that found that tinea capitis is a disease of prepubertal children [3]. This similar finding may be due to poor awareness about hygiene and increased susceptibility to the dermatophyte at younger age because of lower sebaceous gland secretion which may have inhibitory effect for dermatophyte infection in older age. On the other hand, tinea corporis $6.3 \%$ and tinea unguium were more frequent in age group 10-14, within the urban residents and the grade level range of 5-6, and that may be due to increased activities in older age which may transmit fungi like sharing nail clippers, sport wears, and cosmetics use in older age.

Dermatophyte species variation with respect to the above variables was also assessed. There was a statistically significant association between dermatophyte species and children's sex and residence $(p<0.05)$. T. rubrum and T. violaceum were more frequent in urban inhabitants and male sex whereas $M$. canis was prevailing in the suburb or rural condition, as shown in Table 2. Except frequency variation the other variables were not statistically associated $(p>0.05)$. A study among Nigerian school children showed that the most prevalent species among the males was $T$. tonsurans while the least were M. canis and M. gypseum, whereas in females T. tonsurans (25.0\%) and M. canis were equally identified [11]. These variations confirm that the etiologic agents may differ in relation with dermatophyte host preference, occupation, and cultural habits with respect to gender and age of the studied population. Living with domestic animals or farming exposes individuals to zoophilic dermatophytes (M. canis) whereas living in crowded condition may facilitate transmission of anthropophilic dermatophytes (T. rubrum) [2].

\section{Limitation of the Study}

The laboratory analysis was unable to utilise the most recommended dermatophyte test media; therefore, some fastidious dermatophyte fungal species may not have been isolated from clinical specimens. The other limitation of this study is that it shows only temporal association between the studied variables since it is a point prevalence study.

\section{Conclusion}

This study confirmed that the prevalence of dermatophytosis in the studied area was $23.4 \%$ which is similar to other African studies $[5,11,12]$ but in developed countries it was less than $5 \%$ [13] that demonstrates that dermatophytosis is still a common problem in developing countries school children. Tinea capitis was identified as the most prevalent clinical presentation $66.6 \%$ that is similar to the other studies done in other African school children and younger age is the most susceptible group. This study found that $T$. violaceum was the most common etiologic agent followed by T. rubrum but in other studies variable species were identified $[2,7,11,12,14]$ which validates etiologic agent variation with respect to a particular geographical location, socioeconomic status, and life style of the studied population. We recommend further research on the possible risk factors for tinea capitis in the school children.

\section{Competing Interests}

The authors declare that they have no competing interests.

\section{Authors' Contributions}

Alem Alemayehu participated in designing and collecting data and analysed and interpreted data and drafted the manuscript for publication. Gebremedhin Minwuyelet was involved in designing, data collection, and reviewing the initial draft manuscript. Gizachew Andualem conceived, designed the study, and reviewed the initial draft manuscript. All authors read and approved the final manuscript.

\section{Acknowledgments}

The authors would like to thank Haramaya University that provided them with the required budget for this research project. They acknowledge Mr. Nardos Asegid for his contribution during laboratory and field work. 


\section{References}

[1] B. Havlickova, V. A. Czaika, and M. Friedrich, "Epidemiological trends in skin mycosesworldwide," Mycoses, vol. 51, supplement 4, pp. 2-15, 2008.

[2] G. A. Patel, M. Wiederkehr, and R. A. Schwartz, "Tinea cruris in children," Cutis, vol. 84, no. 3, pp. 133-137, 2009.

[3] P. R. Murray, K. S. Rosenthal, and M. A. Pfaller, Medical Microbiology, Saunders, 7th edition, 2013.

[4] M.-P. Hayette and R. Sechelt, "Dermatophytosis trends in epidemiology and diagnostic approach," Current Fungal Infection Reports, vol. 9, pp. 164-179, 2015.

[5] C. Seebacher, J.-P. Bouchara, and B. Mignon, "Updates on the epidemiology of dermatophyte infections," Mycopathologia, vol. 166, no. 5-6, pp. 335-352, 2008.

[6] M. Ameen, "Epidemiology of superficial fungal infections," Clinics in Dermatology, vol. 28, no. 2, pp. 197-201, 2010.

[7] B. L. Hainer, "Dermatophyte infections," American Family Physician, vol. 67, no. 1, pp. 101-109, 2003.

[8] R. M. Andrews, J. McCarthy, J. R. Carapetis, and B. J. Currie, "Skin disorders, including pyoderma, scabies, and tinea infections," Pediatric Clinics of North America, vol. 56, no. 6, pp. 14211440, 2009.

[9] A. Chepchirchir, C. Bii, and J. O. Ndinya-Achola, "Dermatophyte infections in primary school children in Kibera slums of Nairobi," East African Medical Journal, vol. 86, no. 2, pp. 59-68, 2009.

[10] D. Ellis, "Practical Identification of Common Dermatophytes," Mycology Online, http://www.mycology.adelaide.edu.au/.

[11] C. A. A. Pires, N. F. S. da Cruz, A. M. Lobato, P. O. de Sousa, F. R. O. Carneiro, and A. M. D. Mendes, "Clinical, epidemiological, and therapeutic profile of dermatophytosis," Anais Brasileiros de Dermatologia, vol. 89, no. 2, pp. 259-264, 2014.

[12] J. N. Dike-Ndudim, I. Ukogo, K. O. Dike et al., "Fungal agents associated with dermatophytosis among pupils in Isu local government area (L.G.A), Imo State, Nigeria," International Research on Medical Sciences, vol. 1, no. 3, pp. 024-029, 2013.

[13] E. V. Komba and Y. M. Mgonda, "The spectrum of dermatological disorders among primary school children in Dar es Salaam," BMC Public Health, vol. 10, article 765, 2010.

[14] M. Pérez-González, J. M. Torres-Rodríguez, A. Martínez-Roig et al., "Prevalence of tinea pedis, tinea unguium of toenails and tinea capitis in school children from Barcelona," Revista Iberoamericana de Micologia, vol. 26, no. 4, pp. 228-232, 2009.

[15] E. I. Nweze, "Dermatophytosis among children of Fulani/Hausa herdsmen living in southeastern Nigeria," Revista Iberoamericana de Micologia, vol. 27, no. 4, pp. 191-194, 2010. 

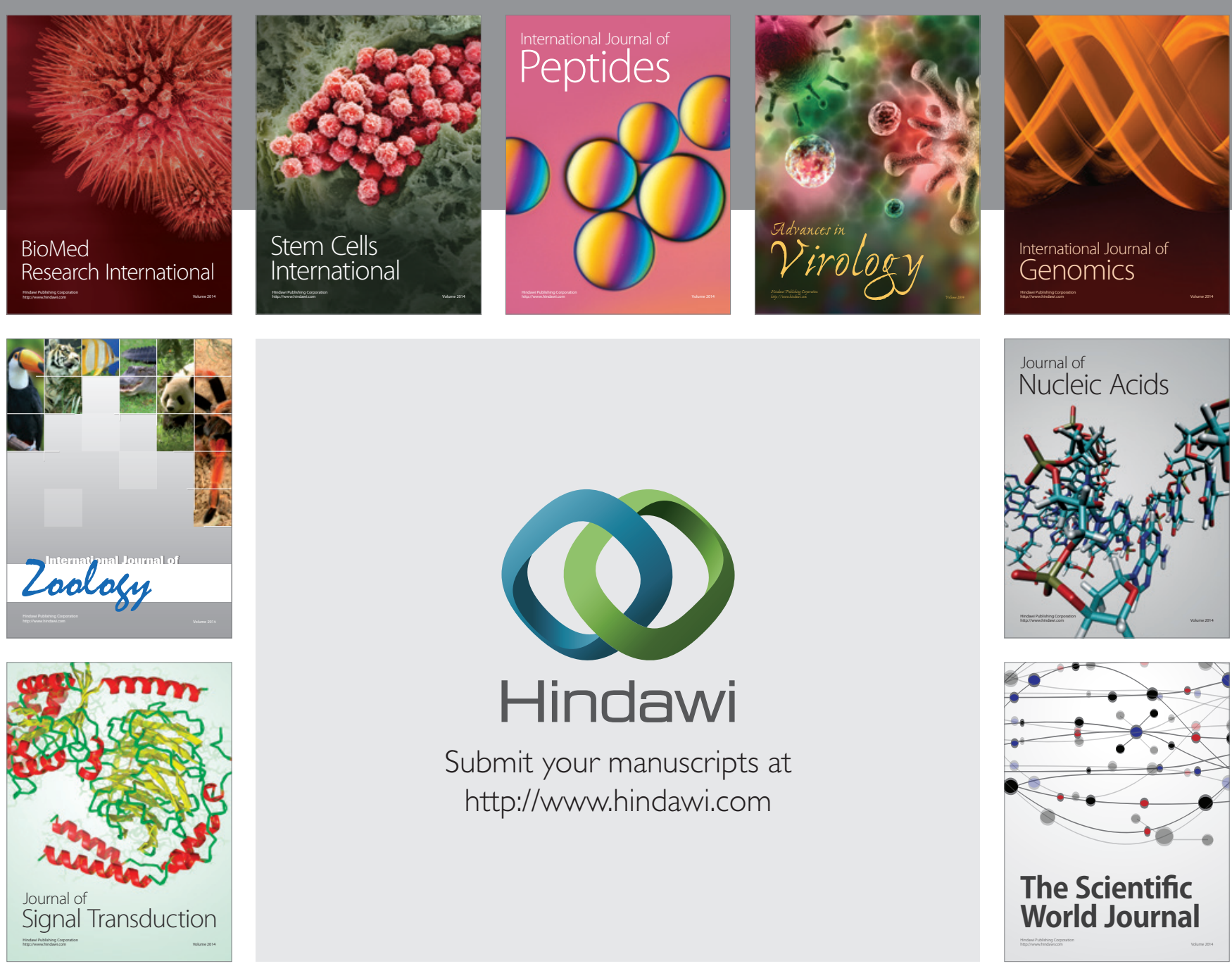

Submit your manuscripts at

http://www.hindawi.com
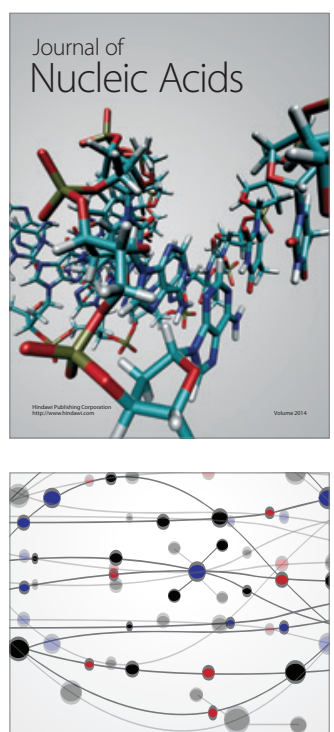

The Scientific World Journal
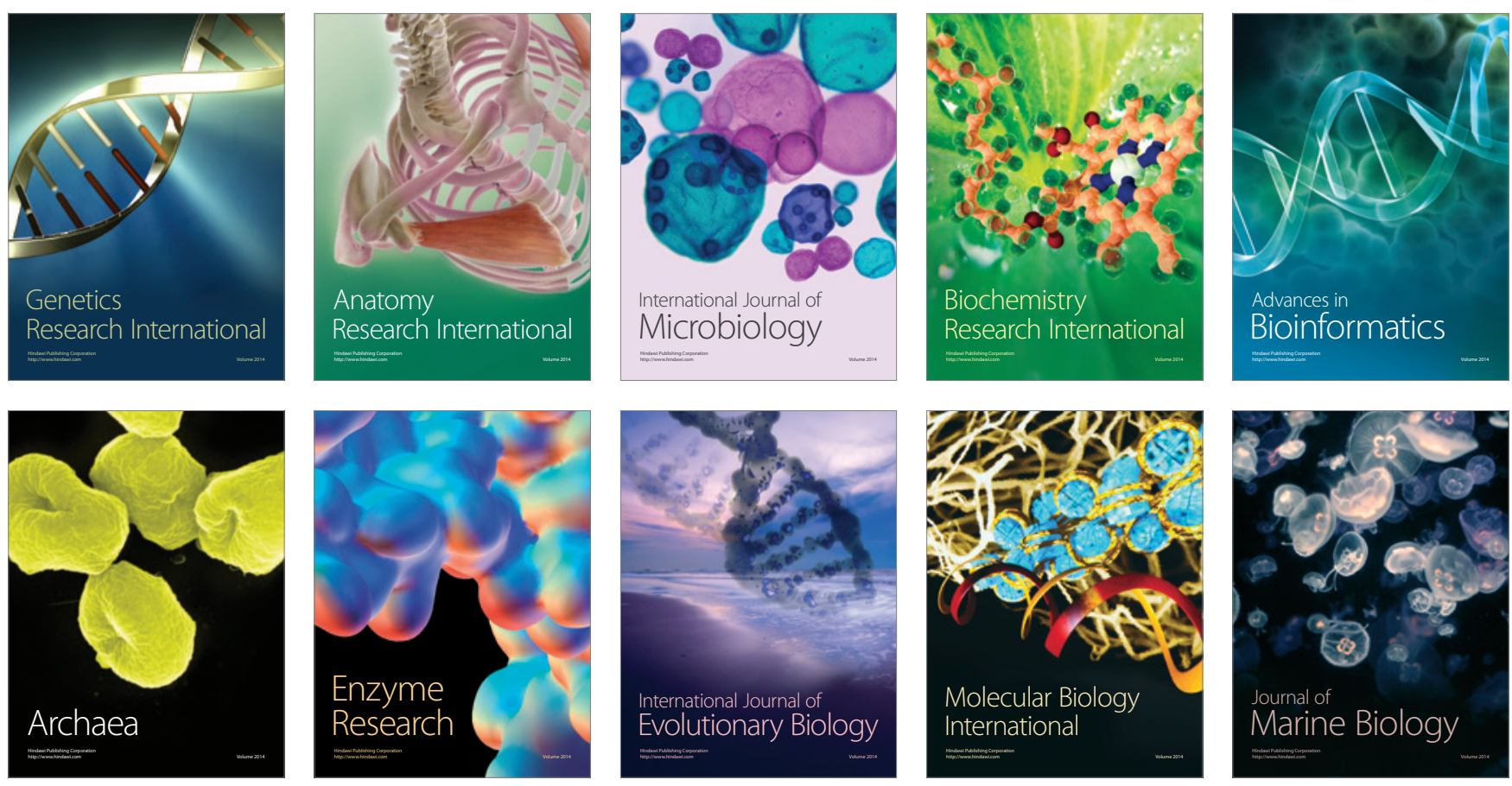